Monatsschrift für Geburtshülfe u. Gynäkologie 1909;30:I-VI

\title{
Contents, Vol. 30, 1909
}

\section{Inhalts -Verzeichnis.}

Original-Arbeiten.

Adolpli, Jos., Die Bedeutung des Levatorspaltes für die $\mathrm{P}^{1 / 8} / 8$ ezidivprognose der Prolapsoperation 320

Boxer, Siegfried, Beitrag zur Kenntnis des Tubenkarzinoms. (Hierzu Tafel VI-VII) 549

Fränkel, L., und L. B ö h m , Genitalblutungen bei

Hämophil·ie 417

Franqué, Otto v., Zur Statistik der operativen Behandlung bei Uteruskarzinomen

Gottschalk, S., Erwiderung auf die epikritischen Bemerkungen Schottländers zu meiner Arbeit ,,Ueber die Entstehung der Erosion der Portio vaginalis" in Bd. XXX, Heft 2 dieser Monatsschrift 339

Himmelheber, K., Zur Bedeutung der glandulären

Hyperplasie und Hypertrophie des Endometriums . . 159 Hoe line, O., Die Beziehungen der Bettruhe nach gynä-

kologischen Operationen und Entbindungen zur Throm-

bose und Embolie $\quad 544$

Kehrer,E., Was leistet die Martinsche Salpingo-Stomato-

plastik? 582

, Zur Kenntnis der desmoiden Geschwülste der Vagina.

(Hierzu Tafel IX) 731

Kuhn, Franz, Steril-Katgut 488

Luft, Heinrich, Luxatio humeri congenita . . . 307 M a r t i n , A., Das Centennarium der Ovariotomie. (Hierzu

Tafel VIII) 675

Natansohn, Karl und Waldemar Goldschmidt, Ueber das morphologisohe Verhalten der

Montgomeryschen Drüsen. (Hierzu Tafel I-II) ... 34 Novak, Josef, Bedeutet der Kaiserschnitt einen Fort-

schritt in der Therapie der Placenta praevia ? .... 458

$-\mathrm{IV}-$

Ogata, J., Untersuchungen über den Hämoglobingehalt und die Anzahl der roten und weissen Blutkörperchen der Wöchnerinnen 706

Pfannenstiel, J., Ueber die geburtshülflichen Hülfsoperationen bei uneröffnetem Muttermund 541 
$\mathrm{R}$ i e c k , A., Transperitoneale vaginale supra vaginale Uterusampufcation und transperitoneale vaginale Uterusexstirpation 724

Rosthorn, Alfons v., Appendicitis und Erkrankungen der Adnexa uteri 280

Scheidler,Friedrich, Zur Kasuistik der Infektionen mit dem Bacillus aërogenes capsulatus in geburtshülflichen Fallen 714

Schiller, H., Seltener Fall von Uterus duplex bicornis . 304

Schottländer, J., Epikritische Bemerkungen zu der

Arbeit Gottschalks: Ueber die Entstehung der Erosion der Portio vaginalis 191

Schultze, B. S., Ueber Querlage, Wendung, Ex-

traktion 145

Sellheim, Hugo, Zwei Erleichterungsmittel der kom-

binierten Untersuchung 587

S o h m a , M., Ueber die Ausscheidung von Antitoxin und

Präzipitinogen durch die Milchdrüse bei passiv immuni-

sierten Müttern 475

Taussig, Fred J., Die Entwicklung des Hymen . . 690

Thorn, W., Zur vaginalen Myomotomie $\quad 570$

W e r t h , Richard, Ueber ein neues Verfahren der supra vaginalen Cervixresektion mit Erhaltung der Portio

vaginalis bei Elongatio colli

1

- -, Ein Fall von erworbener, hochgradiger Stenose des Cervikalkanals, geheilt durch

supravaginale Cervixresektion mit Erhaltung der Portio 153

Sammelreferate.

Bericht über die geburtshülflich-gynäkologische Literatur in

Nord-Amerika. Januar bis Juni 1908. Von A r t h u r

Stein in New York 497

Die klinische Bedeutung der Thrombose und der derzeitige

Stand ihrer chirurgischen Behandlung. Von A d o $1 \mathrm{p} \mathrm{h}$

Hoffmann in Greifswald 738

Der gegenwärtige Stand der operativen Geburtshülfe beim

engen Becken. Von Adolf H ü $\mathrm{f} f$ el in Erlangen . 588

$-\mathrm{V}-$

Typhus xxnd Schwangerschaft. Von L. K n a p p in Prag 43, 208 Ueber die Behandlung der Placenta praevia. Von Josef

Nov a $\mathrm{k}$ in Wien 192,340

$\mathrm{Z}$ u r IV. ï a g u n g der Vereinigung z u r F ö r -derungdesHebammenwesensinStrass-burg i. E. am 1. Juni 1909 .......... 59

Strassburg $1909 \quad .60$

Bericht über die IV. Versammlung der Vereinigung zur Förderung des deutschen

Hebammenwesens in Strassburg und d i e XIII. Versammlung der deutschen Gesellschaft für Gynäkologie in Strassburg v o m 1.-5. Juni 1909. Erstattet von

Eduard Martin in Berlin 
34. Jahresversammlung der amerikani-s c h e n Gesellschaft für Gynäkologie.

Von A r t h u r S t e i n in New York 357

B u d a p e s t 1909. Von A. Martin in Berlin .... 512

XVI. Internationale $\mathrm{r} \mathrm{m}$ edizinischer $\mathrm{K}$ o $\mathrm{n}$ -

g r e s s inBudapes $\mathrm{t} v$ o $\mathrm{m} 28$. August bis

4. S e p t e m b e r 1909. Geburtshülflich - gynäko-

iogische Sektion. Bericht, erstattet von Dr. W e i b e 1

in Wien 513

S a 1 z b u rg 1909. Von F. S c h a u t a in Wien ... 526 Die geburtshülflich-gynäkologische A b -t e i 1 u ii g d e r 81. V e r s a m in lung d e u t s c h e r Nat urfo r s c h e r und A e r z t e in Sa 1zbu rg vom 19. bis 25. September 1909. Bericht, erstattet von A. W a g n e r in Wien ...

Julius v. Elischer† . . . .

605

A. 0 . Lindfors $\dagger \ldots . . .766$

GustavLott $\dagger \quad \ldots \quad 260$

$\mathrm{H}$ e $\mathrm{r}$ in a $\mathrm{n} \mathrm{n} \mathrm{J}$ o h a $\mathrm{n} n$ e s P f a n nenstiel $\uparrow$. (Hierzu

Porträt) 137

A 1 fon s v. Ro sthorn †. Mit Porträt ........ 261

M a x R u n g e †. Mit Porträt 367

Tagesnachrichten und Personalien . ., 106, 366, 511, 606, 767

- VI - Vereins- und Literaturbeilage.

Original-Sitzungsberichte aus geburtshülflieh-gynäkologisehen

Gesellschaften:

Gynäkologische Gesellschaft in Breslau 224, 653

Mittelrheinische Gesellschait für Geburtshülíe und Gy-

kologie114, 607

Münchner Gynäkologische Gesellschaft $\quad 110$

Freie |Vereinigung von Frauenärzten in München $\quad 788$

Niederrheinisch-westiälische Gesellschait für Gynäkologie

und Geburtshülfe $\quad 238,768$

Nordostdeutsche Gesellschait iür Gynäkologie .... 373, 633

Gynäkologische Gesellschait in Wien 379

Referate aus geburtshülflich-gynäkologischen Gesellschaften

$125,242,383,534,660,804$

Geburtshülflich-gynäkologisehe Mitteilungen aus anderen Gesellschaften und Vereinen . 129, 244, 388, 534, 663, 805

Buehanzeigen .... 130, 245, 390, 665, 805

Literaturverzeichnis $131,255,411,535,668,807$

Sachregister 811

Namenregister $\quad 819$ 